Journal of the Electrochemical Society, 1998, Volume 145, Issue 7, Pages 2285-2295.

ISSN: (Print 0013-4651) (Online)

DOI: $10.1149 / 1.1838633$

http://www.electrochem.org/

http://scitation.aip.org/JES

http://scitation.aip.org/getpdf/servlet/GetPDFServlet?filetype=pdf\&id=JESOAN000145000007002285000001\&idty pe $=$ cvips \&prog $=$ normal

(C) The Electrochemical Society, Inc. 1998. All rights reserved. Except as provided under U.S. copyright law, this work may not be reproduced, resold, distributed, or modified without the express permission of The Electrochemical Society (ECS). The archival version of this work was published in Journal of the Electrochemical Society, 1998, Volume 145, Issue 7, Pages 2285-2295.

\title{
Characterization of AA2024-T3 by Scanning Kelvin Probe Force Microscopy
}

\author{
P. Schmutz and G. S. Frankel
}

Fontana Corrosion Center, Department of Materials Science and Engineering, The Ohio State University

\begin{abstract}
Volta potential mapping of AA2024-T3 on surfaces was performed with an atomic force microscope. A linear relation was found between the Volta potential measured in air and the corrosion potential in aqueous solution for a range of pure metal samples, indicating that this potential is a measurement of the practical nobility of the surface. Large differences in the Volta potential of intermetallic particles in AA2024-T3 and the matrix phase resulted in a potential map with high contrast that clearly identifies the location of the particles. All intermetallic particles, including the $\mathrm{Mg}$-containing $\mathrm{S}$-phase particles, had a Volta potential noble to that of the matrix. Surface films on the particles and the matrix were found to have strong effects on the potential, and probably explain the noble nature of the $\mathrm{Mg}$-containing particles, which have been reported to be active to the matrix in solution. The effect of these surface films was examined by refreshing the sample surface using different techniques. Lateral heterogeneities in certain intermetallic particles were also revealed.
\end{abstract}

\section{Introduction}

AA2024-T3 (A1-4.4Cu-1.5Mg-0.6Mn) is a high strength Al alloy that is used extensively in aerospace applications. Unfortunately, it is rather susceptible to localized corrosion in chloride-containing environments as a result of the heterogeneous microstructure that is introduced during solidification from the melt or intentionally developed for optimization of mechanical properties. ${ }^{1}$ The inhomogeneous distribution of $\mathrm{Cu}$ in AA2024-T3 associated with intermetallic particles results in galvanic cells that lead to localized attack. ${ }^{1}$ The importance of the intermetallic particles (especially the copper-containing particles) as initiation sites for corrosion has been addressed in several papers. ${ }^{1-3}$ The corrosion potentials of intermetallic particles have been determined from measurements on specially made bulk samples, ${ }^{4,5}$ although the extent to which these bulk analogs imitate the behavior of the real particles is not clear. Buchheit has compiled the corrosion potentials in solution of different phases present in the Al$\mathrm{Cu}-(\mathrm{Mg}, \mathrm{Fe}, \mathrm{Mn}, \mathrm{Si})$ alloy system, which includes AA2024. ${ }^{6}$ These measurements on bulk intermetallic samples indicate the relative nobility of the two main types of particles in AA2024T3; Al-Cu-(Fe, Mn) particles should be noble relative to the Al matrix, whereas $\mathrm{Al}-\mathrm{Cu}-\mathrm{Mg}$ particles should be active. ${ }^{6}$

The situation in commercial alloys is complicated by the variety of particle distributions that can exist. ${ }^{7}$ It has been shown that the cooling procedure and aging time result in drastic differences in the corrosion behavior. ${ }^{7}$ The exact distribution and size of certain types of 
intermetallic particles as well as the related composition gradient in the matrix around the particles influence later stages of corrosion such as intergranular corrosion ${ }^{8-10}$ and stress corrosion cracking ${ }^{11}$ of this type of alloy.

Pitting of aluminum-based alloys has been studied in detail, ${ }^{7-11}$ but an understanding of the initial stages of corrosion is still lacking, mainly because appropriate techniques have not been available until recently. Localized corrosion in these alloys has been studied by electrochemical measurements in combination with scanning electron microscopy (SEM) and electron dispersive spectroscopy. Blanc et al. used this approach to study the corrosion of AA2024 and pure Al at different applied potentials. ${ }^{2}$ At low potential they found that copper from the intermetallic particle is redeposited on the surface, and that these sites dominate the corrosion processes. The pit nucleation rate was found to be much higher than for pure $\mathrm{Al}$ in the low potential region. At a higher potential where $\mathrm{Cu}$ is not redeposited, the pit nucleation rate is lower, and the behavior of the alloy is similar to pure Al. The corrosion behavior of AA2024 also changed in sulfate solutions, which favor copper corrosion, compared to chloride solutions, which increase aluminum corrosion.

The mechanism responsible for redistribution of copper on a corroding surface to distances remote from localized intermetallic particles at potentials lower than the reversible potential of copper is not clear yet. Dealloying by preferential dissolution of aluminum or magnesium could be responsible for enrichment of copper on the surface on the intermetallic particle. ${ }^{12}$ However, the simultaneous dissolution of both $\mathrm{Al}^{3+}$ and $\mathrm{Cu}^{2+}$ ions followed by reduction of copper seems unlikely at the corrosion potential. Mazurkiewicz investigated the corrosion of $\mathrm{Al}_{2} \mathrm{Cu}$ with a rotating disk electrode and found no copper in solution. ${ }^{4}$ Recently Buchheit et al. described how dealloying of relatively active S-phase particles (Mg-containing) might result in the formation of a porous copper surface layer, which could lead to a loss of electrical contact with the matrix or nonfaradaic release into solution of very small $\mathrm{Cu}$ particles. ${ }^{1}$ Once isolated from the $\mathrm{Al}$ matrix, the $\mathrm{Cu}$ particles could achieve their own corrosion potential, dissolve into solution, and then redeposit onto the $\mathrm{Al}$ matrix, which is at a lower potential.

In the present study, Volta potential and topographic maps are measured simultaneously with submicron resolution using scanning probe microscopy (SPM). The intermetallic particles can be clearly identified in the potential distribution before any effect of corrosion on the surface topography is detected, and the evolution of the potential and topography after solution exposure can be followed. Furthermore, the Volta potential measurement provides an indication of the nobility of each particle relative to the matrix, which is important in determining their role in galvanic interactions.

The Kelvin probe technique has been used for some time to measure the potentials of various metal surfaces by nulling the current flowing between the sample and a closely positioned probe electrode. ${ }^{13}$ In 1979, Hoelzl and Schulte published a compilation of the work functions in vacuum determined by the Kelvin probe technique for most of the common pure elements and for adsorption processes. ${ }^{14}$ The influences of internal stresses and temperature on the work function were discussed. More recent studies have investigated model electrochemical interfaces in vacuum. ${ }^{15-17}$ Stratmann has demonstrated the principle ${ }^{18,19}$ and the utility of the Kelvin probe technique in corrosion studies of iron ${ }^{20,21}$ and aluminum. ${ }^{22}$ He measured the Volta potential of samples covered with a thin layer of electrolyte using a probe that did not touch the electrolyte. The Volta potential was shown to vary linearly with the corrosion potential of the sample/solution interface determined with a standard reference electrode positioned in the electrolyte layer. ${ }^{19,20}$ The corrosion potential under an organic coating can be determined, which 
provides information on defects at the metal-coating interface. The potential of a surface can be mapped at a lateral resolution of around $100 \mu \mathrm{m}$ using a scanning Kelvin probe. ${ }^{19}$ The behavior of an artificial galvanic couple (for example $\mathrm{Zn}$ or Pt dots imbedded in an iron matrix) was investigated to check the lateral resolution and to study localized corrosion mechanisms. ${ }^{20}$

The development of the atomic force microscope (AFM) and associated SPM techniques has enabled potential mapping of submicron structures. The approach was developed at the IBM Watson Research Center, where it was used to study photoresist-covered Si wafers ${ }^{23}$ and semiconductor dopant profiles. $^{24}$ Only a few other studies using this technique have been reported. ${ }^{25-30}$ The ability to map the potential on a submicron scale is extremely useful in studies of localized corrosion of Al alloys, which contain heterogeneities on that scale and larger. Furthermore, it may be considered that the distribution of potential across a surface is even more relevant to the corrosion process than surface composition, which can be determined by a range of surface analytical techniques.

\section{Experimental}

Scanning Kelvin probe force microscopy was performed with a commercial AFM (Nanoscope IIIa, Digital Instruments). This instrument can measure the surface topography and potential distribution simultaneously on a line-by-line basis using metal-coated silicon tips that are electrically conducting. The cantilevers were obtained from Digital Instruments, and were coated with a thin layer of Co-40Cr. The topographic profile is first determined along a line using the tapping mode of operation. The tip is then lifted off the surface by $50 \mathrm{~nm}$, and the line is rescanned with the tip maintained at that exact distance at every point along the line by turning off the feedback loop of the AFM and applying the recorded signal from the topographical profile to the scanning piezoelectric element. The potential is determined during the second scan during which an ac voltage $( \pm 1 \mathrm{~V})$ is applied to the tip in order to create an oscillating dipole at the tip. In the presence of an external electric field this dipole will induce an oscillation of the cantilever, which is detected by the normal AFM detection scheme. At each point along the line rescan, a dc voltage ramp is added to the ac signal and the dc voltage that nulls out the cantilever oscillations is determined. At this point, the potential on the tip and the sample are the same. A map of the relative potential of the surface with respect to that of the CoCr tip is determined. This approach to potential distribution measurement is not possible in an aqueous solution because the large voltage applied to the tip would result in faradaic reactions. In this study, all potential mapping was performed in air.

Since this technique is a nulling method and applies a voltage to the tip, the output signal from the instrument was inverted. It is shown below that inversion of the signal was needed to obtain the expected polarity of the potential measurements. The values obtained are also relative to the potential of the $\mathrm{CoCr}$ tip. The $\mathrm{CoCr}$ tips are only pseudo-references since their potential may vary with changes in the surface oxide. However, consecutive measurements of an Al sample in laboratory air with different tips indicated a variation in the Volta potential of $\mathrm{Al}$ of less than $50 \mathrm{mV}$. Nonetheless, in order to avoid errors associated with variations in the tips or instabilities in the instrument electronics, the potential measurements were calibrated by comparison to the potential measured on a pure $\mathrm{Ni}$ surface after immersion in $\mathrm{DI}_{2} \mathrm{O}$. Ni was chosen as a reference because it was found to have a stable potential. All potential measurements are reported herein relative to $\mathrm{Ni}$.

A Philips Electronics model XL-30 scanning electron microscope with a field emission 
gun (FEG) was used. It is equipped with an EDAX Corporation model DX-4 system for energy dispersive spectroscopy (EDS) analysis, and has a superultrathin window for higher sensitivity. An accelerating voltage of $20 \mathrm{kV}$ was used for both secondary electron imaging and EDS.

The AA2024-T3 samples (nominal composition $4.9-3.8 \% \mathrm{Cu}, 1.8-1.2 \% \mathrm{Mg}, 0.9-0.3 \%$ $\mathrm{Mn}, 0.5 \% \mathrm{Fe}$ and $\mathrm{Si}, 0.25 \% \mathrm{Zn}, 0.1 \% \mathrm{Cr}, 0.05 \mathrm{Ti}$, balance $\mathrm{Al}$ ) were wet-ground through 600 grit $\mathrm{SiC}$ paper, and then polished to $1 \mu \mathrm{m}$ in a nonaqueous slurry (Blue Lube from Struers) to minimize corrosion. They were then cleaned in isopropyl alcohol (IPA).

The pure metal samples used for calibration were polished through 1200 grit $\mathrm{SiC}$ paper in water and ultrasonically cleaned in distilled water. The open-circuit potential was measured vs. a standard calomel electrode (SCE).

\section{Results and Discussion}

Calibration of the measurement.-In order to determine the usefulness of the scanning Kelvin probe force microscopy technique, comparisons must be made to potentials measured in bulk solution. Samples were immersed for $30 \mathrm{~min}$ in either $\mathrm{DI} \mathrm{H}_{2} \mathrm{O}$ or $\mathrm{NaCl}$ solution, and a measurement was made of the stabilized open-circuit potential vs. an SCE reference electrode. The samples were then removed from solution, rinsed with DI water, and air dried prior to analysis in the Kelvin probe force microscope. Two sets of potential measurements were performed for each solution and the average values are shown in Fig. 1. The $\pm 100 \mathrm{mV}$ error bars take into account the maximum spread of the values. The Volta potential measured by the Kelvin probe force microscope is seen in Fig. 1 to vary linearly with the open-circuit potential measured previously in solution. The potential values measured with the AFM are given relative to the potential of $\mathrm{Ni}$. As stated above, in order to obtain the relation in Fig. 1, the sign of the potential values determined by the AFM had to be inverted. In this paper, all reported values of Volta potential are inverted and given relative to the potential of $\mathrm{Ni}$.

The potential at any charged interface can be separated into two components: the outer potential (or Volta potential) $\Psi$ and the surface potential $\chi \cdot \cdot^{13,31}$ The outer potential is the electrostatic work done to bring a charge from infinity to just outside the sample where image charges just begin to be important. ${ }^{13}$ The surface potential is associated with surface dipoles and can only be accessed by completely crossing the interface. For a metal surface in an environment other than vacuum, the outer potential of the liquid or gas phase must be considered, resulting in the outer potential difference or the Volta potential. ${ }^{13}$ Textbook descriptions indicate that measured Volta potentials are constant over a wide distance range (from $100 \mathrm{~nm}$ to a few microns away from the surface), but should drop off for measurements made at distances less than about $100 \mathrm{~nm}$ owing to the effect of image charges at the surface electrode $\chi_{\cdot}^{13,31}$ In the present study, the tip/surface separation was varied from 50 to $300 \mathrm{~nm}$, and no effect on the measured potential was observed. Nonetheless, it is our belief that the Volta potential is determined by this technique and the distance of 50-300 $\mathrm{nm}$ is in the constant potential region. One explanation is that the tip-sample interaction is integrated over the whole tip area. Most of the tip would be in the constant potential region even if the end were located at $50 \mathrm{~nm}$ from the surface. 

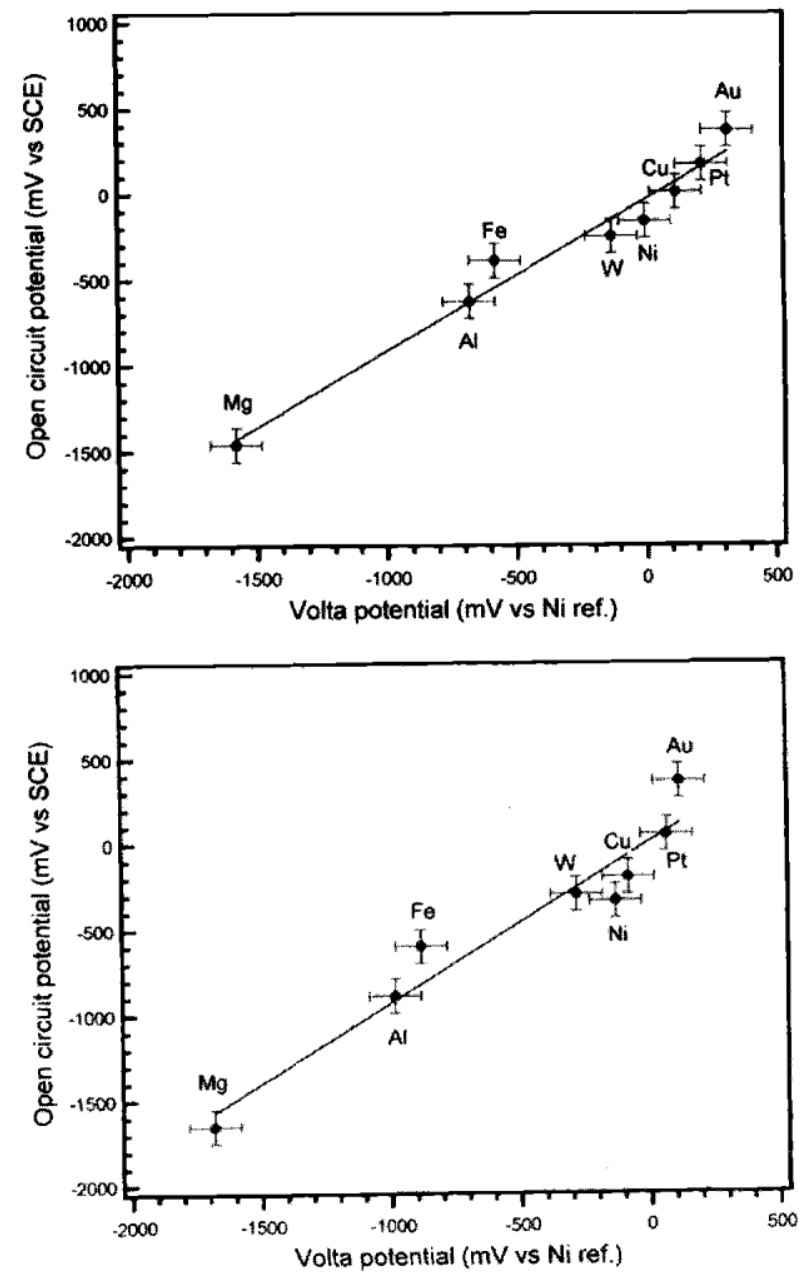

Fig. 1. Comparison of the potential measured in air by Kelvin probe force microscopy with open-circuit potentials measured in solution for different metals in: (a, top) deionized water, (b, bottom) $0.5 \mathrm{M} \mathrm{NaCl}$.

Stratmann has reported a strong linear correlation between the Volta potential measured with the Kelvin probe method above a sample covered with a thin layer of electrolyte and the corrosion potential determined by a reference electrode in the electrolyte layer. ${ }^{19,20}$ In that work the potential of the same interface was determined by two different methods. In Fig. 1, the Volta potential measured in air is compared to the corrosion potential measured in solution. The sample surface and interface region is certainly different in air than in solution. Laboratory air at ambient temperature and relative humidity will result in a few monolayers of adsorbed water on the surface. Experiments carried out by Weaver et al. ${ }^{15}$ and Trasatti ${ }^{17}$ have shown that for noble metals exposed to different solvents in vacuum (simulated electrochemical interface), the measured potential varies quickly for the early stage of adsorption but then is constant after the adsorption of one to two monolayers. Furthermore, Kelvin probe measurements on emersed electrodes have indicated that the double-layer structure developed by applied potential during immersion is maintained for short times when the sample is removed from solution. ${ }^{32,33}$

The linear relation between the open-circuit potential and Volta potential is interesting given that the environments are different. For instance, some of the metals studied, such as Fe and $\mathrm{Cu}$, might have a surface film in air that is very different than that in solution. However, the 
Volta potential measured on Fe in the present work following emersion from water is relatively active, suggesting that a strongly protective film had not yet formed. Kelvin probe measurements of $\mathrm{Fe}$ in gaseous environments were made by Yee et al. ${ }^{19}$ The Volta potential was found to be relatively low under a thin layer of water and stayed low as that water layer evaporated in a dry $\mathrm{N}_{2}$ atmosphere. The Volta potential of this sample then increased during subsequent exposure to $\mathrm{O}_{2}$ with $98 \%$ relative humidity as a result of passivation, and then decreased during subsequent drying in dry $\mathrm{O}_{2}$. However, the detailed dependence of Volta potential on $\mathrm{RH}$ and $\mathrm{O}_{2}$ pressure was not determined. The low Volta potential for Fe observed in the present work is not inconsistent with the observations of Yee et al. of Fe in $\mathrm{N}_{2}$ with low RH. The behavior of Au and Pt electrodes is also of interest because their open-circuit potentials in water are ill-defined, and may be determined by impurities in the system. However, their Volta potentials in air after emersion would be under similar influences. The slope of the line fitted to the data after exposure of the samples to deionized water is 0.9 rather than the slope of 1.0 determined by Stratmann, ${ }^{19}$ likely because of differences in the surface in water and in air.

The open-circuit potentials in $0.5 \mathrm{M} \mathrm{NaCl}$ (Fig. lb) show the same tendency as in distilled water, except that the values are shifted in the active direction by around $200 \mathrm{mV}$ Interestingly, both the open-circuit potential in salt solution and the Volta potential in air following emersion are shifted relative to the values measured in and following DI water exposure, respectively. There is also a larger spread of the data around the line of 0.94 slope, which is likely related to the varied effects of chloride on different metal surfaces.

The values of Volta potential of the samples exposed to air have been found to be unrelated to the work functions of the different metals in vacuum. ${ }^{14}$ Rather, they reflect a modification of the metal work function in a fashion that seems to be similar to that in the presence of a bulk liquid. It should be clear that specific conditions in certain electrolytes might invalidate the predictions made by measurements in air. Nonetheless, the correlation in Fig. 1 suggests that the Volta potential determined by scanning Kelvin probe force microscopy in air following emersion is a measure of the practical nobility of the surface in solution.

Characterization of the intermetallic particles in AA2024-T3.- One feature of the Volta potential measurement made with the AFM is the superior lateral resolution. Figure 2 shows the topography and potential distribution obtained simultaneously on a sample of AA2024-T3 in air. The sample was polished without exposure to water in the final stages, and then only cleaned by a jet of Ar before measurement to avoid further water or alcohol exposure. As a result, many contamination particles remained on the surface, as is visible in the topography scan in Fig. 2. A few raised regions that are barely visible in the surface topography are associated with intermetallic particles that protrude slightly from the surface because of their higher hardness and lower rate of polishing relative to the matrix. In contrast, these intermetallic particles are very clearly distinguished in the Volta potential map on the right side of Fig. 2. In fact, particles that are not evident at all in the surface topography are seen with sharp contrast in the potential map, so the Volta potential is the only way in this case to find intermetallic particles with the AFM. The intermetallic particles have a maximum size of approximately 15 to $20 \mu \mathrm{m}$ and a higher potential than the alloy matrix.

Figure 3 is an SEM image of the same area shown in Fig. 2. Good contrast is obtained because of a difference in scattering of the electrons in the matrix and the particles; Fig. 2 proves that the contrast is not caused by surface topography. There is an exact correlation of the features in the SEM image and the high potential spots in the Volta potential map in Fig. 2. EDS analysis 
performed on different particles confirmed that the regions of higher potential are associated with intermetallic particles. The EDS data are not quantitative because of the small size of the particles relative to the analyzed area (typically $4 \mu \mathrm{m}$ laterally and in depth), but this measurement allows generic identification of the particles as either $\mathrm{Al}-\mathrm{Cu}-(\mathrm{Fe}, \mathrm{Mn})$ or $\mathrm{Al}-\mathrm{Cu}-$ Mg. In Fig. 3, the particles labeled 1-4 are Al-Cu-(Fe, Mn) phases, and the particles labeled A-C are $\mathrm{Mg}$-containing intermetallics. In general (but not always), the $\mathrm{Al}-\mathrm{Cu}-\mathrm{Mg}$ particles are round and smaller than the $\mathrm{Al}-\mathrm{Cu}-(\mathrm{Fe}, \mathrm{Mn})$ particles, which are block shaped. The $\mathrm{Mg}$-containing particles were likely S-phase particles, $\mathrm{Al}_{2} \mathrm{CuMg}$, which is known to be prevalent in AA2024T3. ${ }^{1}$ This could not be confirmed, however, given the uncertainties in the compositions obtained with EDS measurements.

The potential of the matrix (relative to $\mathrm{Ni}$ ) is around $-780 \mathrm{mV}$ in this AA2024-T3 alloy. Differences in the Volta potential of the intermetallic particles relative to the matrix phase create the high contrast in the potential maps. Figure 4a shows a cross section of the Volta potential of particle 1. The Al-Cu-(Fe, Mn) particle has a potential $300 \mathrm{mV}$ more noble than the matrix. A similar difference was reported for the corrosion potential relative to the matrix of a bulk precipitates in a chloride solution, Table I. ${ }^{6}$ A large range of values was reported in that work for the aluminum matrix depending on small variations of $\mathrm{Cu}$ or $\mathrm{Mg}$ content. In Table I, the whole range of potential is shown for the matrix of the AA2024-T3 alloy. As seen in Fig. 4a, the center of the particle is even more noble than the rest of the particle. This region corresponds exactly to the spot analyzed by EDS. The ennoblement of the area is likely an artifact resulting from the deposition of carbon contamination on the surface during the EDS analysis. This indicates how sensitive the scanning Kelvin probe force microscopy technique can be to the surface condition.

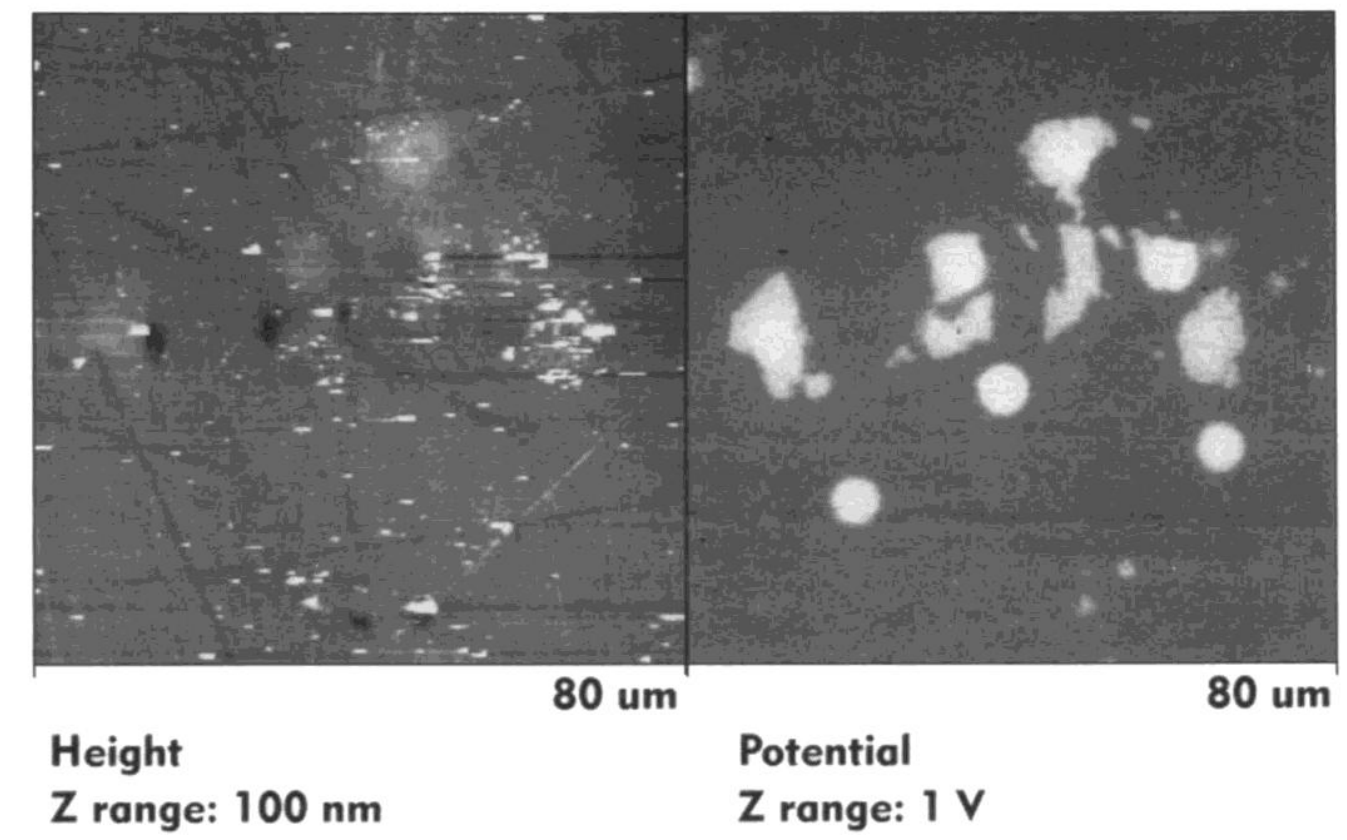

Fig. 2. AFM image of intermetallic particles in an AA2024-T3 alloy. On the left, a topographical map of the surface, and on the right, the Volta potential distribution of the same area.

The Al-Cu-Mg particles have a Volta potential of about $-500 \mathrm{mV}$ relative to $\mathrm{Ni}$, which is slightly lower than that of the Al-Cu-(Fe, Mn) particles. There was no indication of a more-noble 
contaminated area on the $\mathrm{Al}-\mathrm{Cu}-\mathrm{Mg}$ particles studied, but it cannot be excluded that some contamination was present as a result of the SEM investigation. Considering that the potential increase associated with carbon contamination on particle 1 was $100 \mathrm{mV}$, it is possible that the potential of the $\mathrm{Al}-\mathrm{Cu}-\mathrm{Mg}$ particles was only about $-600 \mathrm{mV}$ relative to $\mathrm{Ni}$. Even so, it would still be noble compared to the $\mathrm{Al}$ matrix. This nobility in air of the $\mathrm{Al}-\mathrm{Cu}-\mathrm{Mg}$ particles relative to the matrix is in contrast to the corrosion potential of a bulk Al-Cu-Mg sample in a bulk aqueous solution, which was found to be active relative to the matrix. ${ }^{6}$ It is believed that the high potential on the Mg-containing particles in air is caused by a surface film of altered composition compared to the bulk of the particles. This altered layer certainly contains a surface oxide film, but may also include gradients in composition in the oxide or underlying metal that may result from preferential corrosion of certain elements even in the nonaqueous polishing slurry. Exact determination of the composition and structure of this layer will require more detailed surface analysis. The presence of this surface film has strong influences on the behavior of these particles upon immersion of the alloy in a chloride solution as will be reported in a separate communication. $^{34}$

The Volta potential of $-780 \mathrm{mV}$ measured for the matrix of AA2024-T3 alloy is lower than the potential of $-680 \mathrm{mV}$ measured on a sample of pure Al after water exposure, see Fig. la. The reason for this lower potential of the AA2024-T3 is not clear. Exposure of the AA2024-T3 sample to distilled water did not change the measured Volta potential.

Another aspect to be addressed when characterizing the Volta potential of a sample is the possible influence of the topography. Figure 5 shows that there is no potential difference associated with the hole on the left side of the topographic map, but the one on the right exhibits a noble potential. From this example, it is obvious that a topographical feature like a hole does not automatically produce a potential difference. In general, even extremely rough surface features on pure aluminum, copper, or magnesium samples do not generate a significant potential contrast. On the other hand, it is not clear why some holes with very noble potential are present. Even samples polished with great care in nonaqueous polishing media and not further exposed to corrosive conditions show this kind of hole at the border of some intermetallic particles (Fig. 2 and 3).

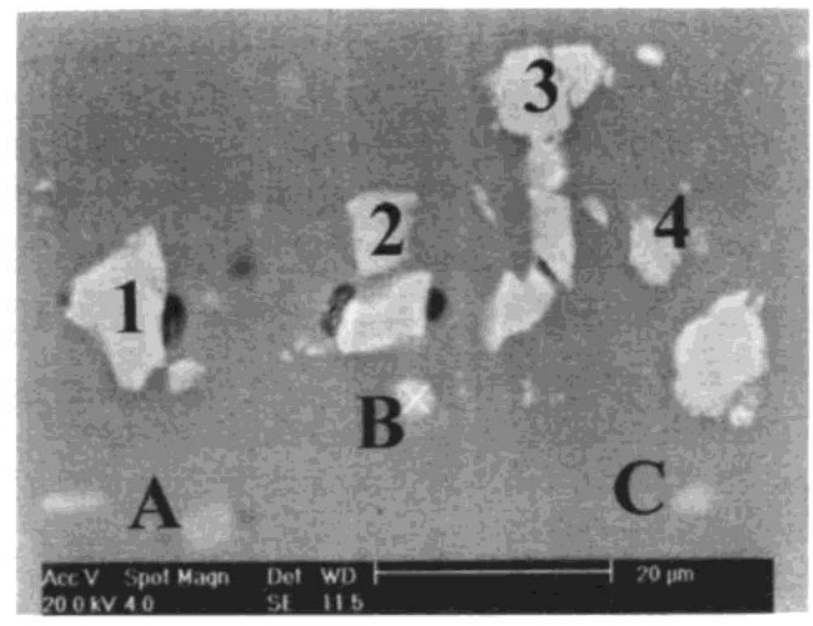

Fig. 3. SEM image of the same area as Fig. 2. EDS analysis indicated that particles 1-4 are $\mathrm{Al}-\mathrm{Cu}-(\mathrm{Fe}, \mathrm{Mn})$ intermeallics and $\mathrm{A}, \mathrm{B}$, and $\mathrm{C}$, are $\mathrm{Al}-\mathrm{Cu}-\mathrm{Mg}$ intermetallics. 

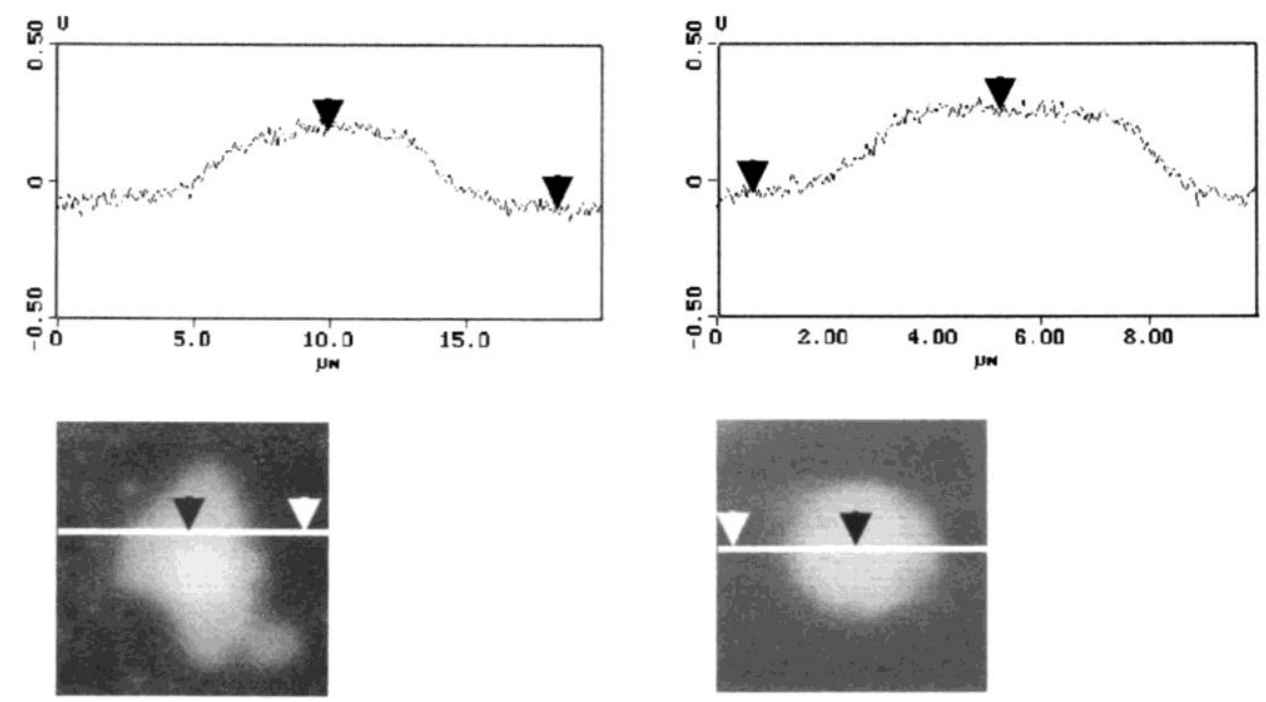

Fig. 4. Potential profiles across (a, left) particle 1 of Fig. 3 and (b, right) particle B of Fig 3.

Normal and lateral gradients of Volta potentials associated with intermetallic particles.-As described above, the altered surface layer on the Mg-containing particles results in a noble Volta potential in air relative to the matrix. In order to investigate the influence of surface films, several techniques were used to refresh the metal surface. A silicon tip was rastered in air on a portion of the same sample area shown in Fig. 2 and 3. This rastering was performed in the contact mode of the AFM with a relatively high tip force in order to remove part of the surface layer. About 2-3 nm were removed as a result of the abrasion, and the debris generated was dragged to the edge of the rastered area, Fig. 6. The whole rastered area (both matrix phase and intermetallic particles) had a Volta potential about $100 \mathrm{mV}$ more active (lower potentials) than similar regions that were not abraded. The surface layer on the particle may contain both an oxide and a dealloyed layer, whereas the film on the matrix is likely only an Al oxide, with no dealloyed layer. It could be that the original altered surface layer on the particles and matrix was thicker than 2-3 nm, and that the abrasion did not penetrate to the bulk material. Even if it did, a new oxide would immediately form. It is possible that physical processes associated with abrasion, such as plastic deformation, caused the Volta potential to become more active in the abraded area. However, it is more likely that the surface layer (including oxide and perhaps a dealloyed layer) on the particles and matrix was partially or totally removed by the rastering, exposing a new surface that was less noble than the original surface.

A 2-3 $\mathrm{nm}$ deep scratch was made into one of the $\mathrm{Mg}$-containing particles in this area. A magnified view of this particle is shown in Fig. 7. The scratch is clearly seen in the topographic map on the left. The corresponding region in the Volta potential map has a yet lower value than the rest of the particle, which itself was made more active by the rastering. Figure 8 shows a potential profile across part of the rastered and scratched area. The scratch corresponds to the low potential peak that is seen in the potential profile of the intermetallic particle. The potential at the bottom of the scratch is almost the same as in the rastered aluminum matrix and lower that in the unrastered area. This is an indication that there is a depth distribution of the potential in the first few nanometers of the surface and also that the Volta potential in air on the $\mathrm{Al}-\mathrm{Cu}-\mathrm{Mg}$ intermetallic particle can be more active than the matrix under certain conditions.

In another attempt to investigate this depth distribution, the surface was sputter-etched 
with an argon plasma. Figure 9 shows the potential map for the area shown in Fig. 2 after sputter-etching to a depth of approximately $10 \mathrm{~nm}$. Comparison of the potential maps in Fig. 2 and 9 reveals that the potential contrast of the three $\mathrm{Mg}$-containing particles (labeled A-C in Fig. 3) disappeared after sputter-etching. The topographic maps (not shown) indicate that the particles themselves have not been etched away. However, they have the same potential as the matrix, even with the newly formed surface oxide film that must be present. These Mg-containing particles may dissolve actively with no oxide film in a chloride solution. The observation that the Volta potential in air of a freshly scratched region of such a particle is close to that of the Al matrix supports the observation mentioned above that the corrosion potential of these $\mathrm{Mg}$ containing particles is lower than that of the matrix in solution. ${ }^{6}$

In order to investigate longer term aging of these surfaces, another sample was sputter-etched in the same fashion as the previous sample, stored in laboratory air for 2 months, and then reexamined. Figure 10a shows the potential distribution of an area immediately after sputter etching. A backscattered electron image of the same region after sputter etching is seen in the white box in Fig. 10b. The large Mg-containing particles in this image are labled A-C. These particles were clearly observable in the Volta potential map prior to sputter etching (image not shown). However, as shown above, after etching they are not visible in the potential map because their potential contrast has been removed by the etching. Figure 10c shows the potential map of the same area 2 months after sputter etching. The Mg-containing particles are still not visible. Whatever oxidation that may have occurred did not result in an increase in the potential of the particles. Therefore, the noble potential of the Mg-containing particles in the as-polished condition may be associated with selective dissolution during the polishing procedure, even though a nonaqueous polishing slurry was used.

Table I. Comparison of Volta potentials measured in air and corrosion potentials in $0.5 \mathrm{M} \mathrm{NaCl}$ obtained from the literature. ${ }^{6}$

\begin{tabular}{lcc}
\hline & $\begin{array}{c}\text { Volta potential in air } \\
\text { (mV vs. Ni) }\end{array}$ & $\begin{array}{c}\text { Corrosion potential } \\
\text { (mV vs. SCE) }\end{array}$ \\
\hline $\mathrm{Al}$ matrix & -780 & -880 to 610 \\
$\mathrm{Al}_{2} \mathrm{CuMg}$ & $-500 \pm 50$ & -920 \\
$\mathrm{Al}_{2} \mathrm{Cu}(\mathrm{Fe}, \mathrm{Mn})$ & $-480 \pm 50$ & -675
\end{tabular}




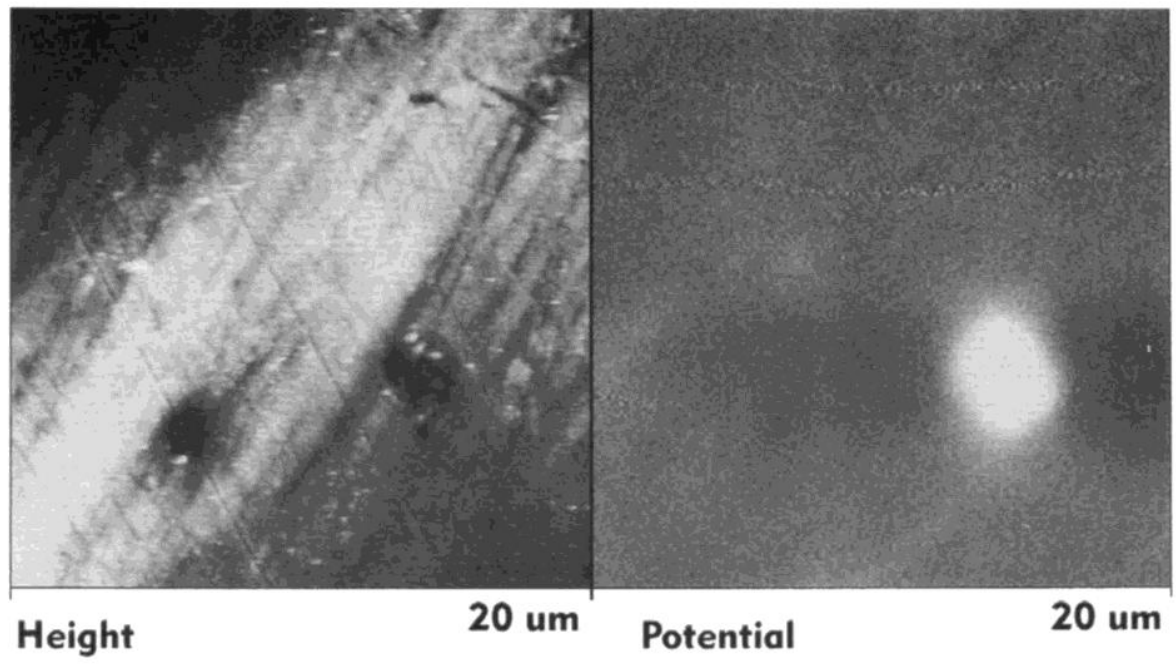

$Z$ range: $200 \mathrm{~nm}$

$\mathrm{Z}$ range: $1.5 \mathrm{~V}$

Fig. 5. Topographic and potential maps of an area containing two holes.

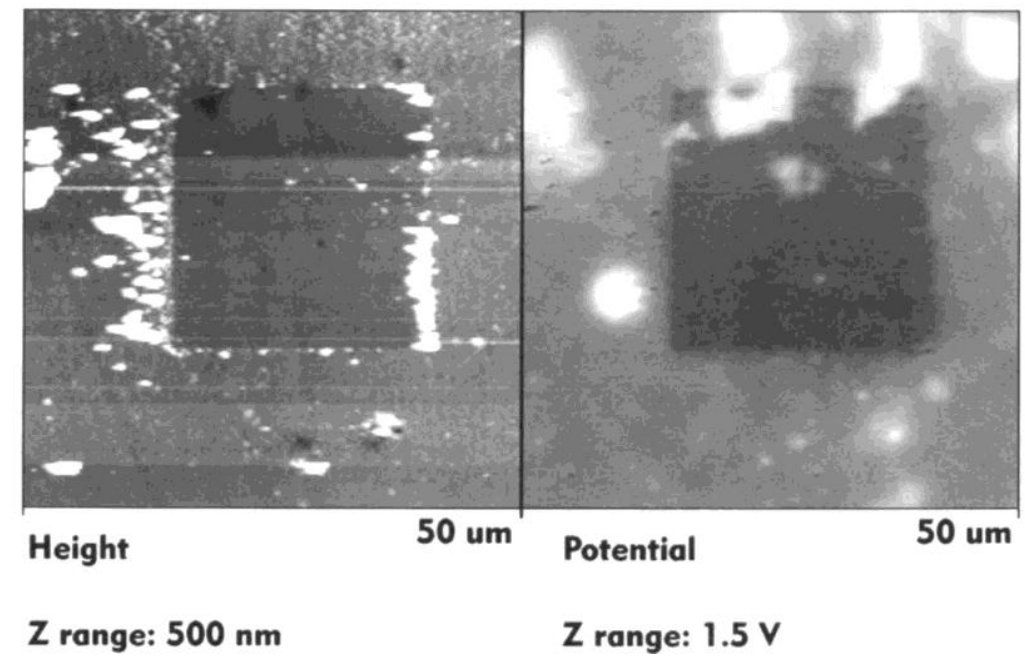

Fig. 6. Enlargement of the region presented in Fig. 2 and 3 after rastering a $25 \mu \mathrm{m}$ square central area in air at high force and scratching particle B as labeled on Fig. 3.

After removing the first few nanometers on the surface of a sample by sputter etching, it is possible to see a lateral distribution of the potential on certain particles, Fig. 11. Many noble spots (micron or smaller in size) become visible after sputter-etching. In the center of this figure there is a large $\mathrm{Al}-\mathrm{Cu}-(\mathrm{Fe}, \mathrm{Mn})$ intermetallic particle with a distinct region of different potential. Figure 12 shows a backscattered electron image generated in a SEM on the same type of particle as in Fig. 11. The bright edges indicate that relatively heavy elements (e.g., $\mathrm{Fe}, \mathrm{Mn}, \mathrm{Cu}$ ) are enriched. Elemental segregation within the particles is consistent with the variation of potential observed in Fig. 11 in certain regions. It should not be surprising that the intermetallic particles are not homogeneous in nature since they may be formed interdendritically during solidification from the melt. This inhomogeneity can have strong implications in the behavior of the particle and matrix upon immersion in a corrosive environment. 


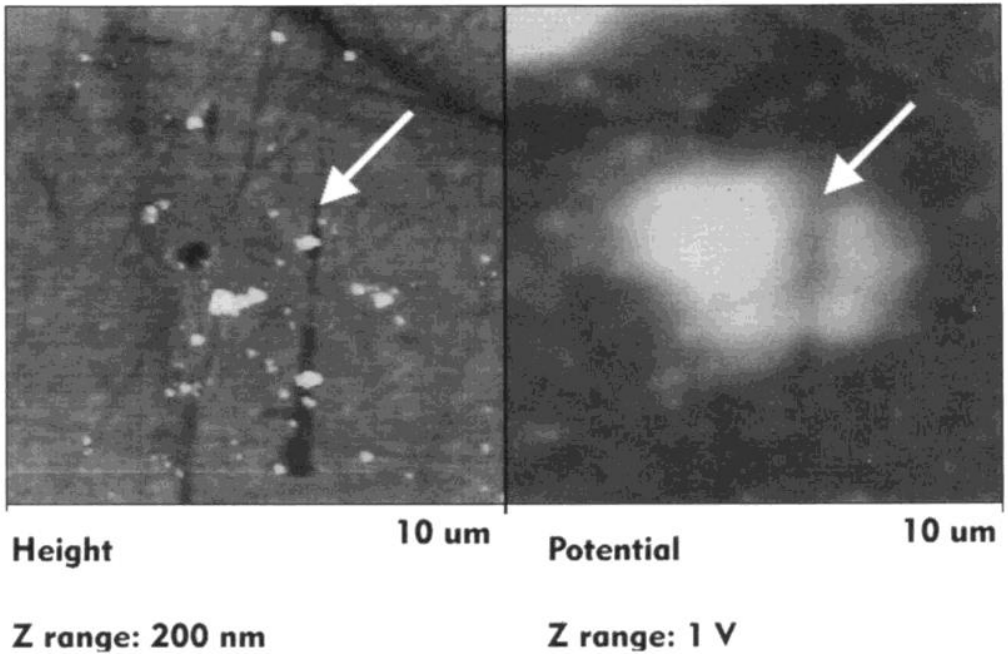

Fig. 7. Detail of the scratched particle at the center of Fig. 6. The arrows indicate the scratch location.
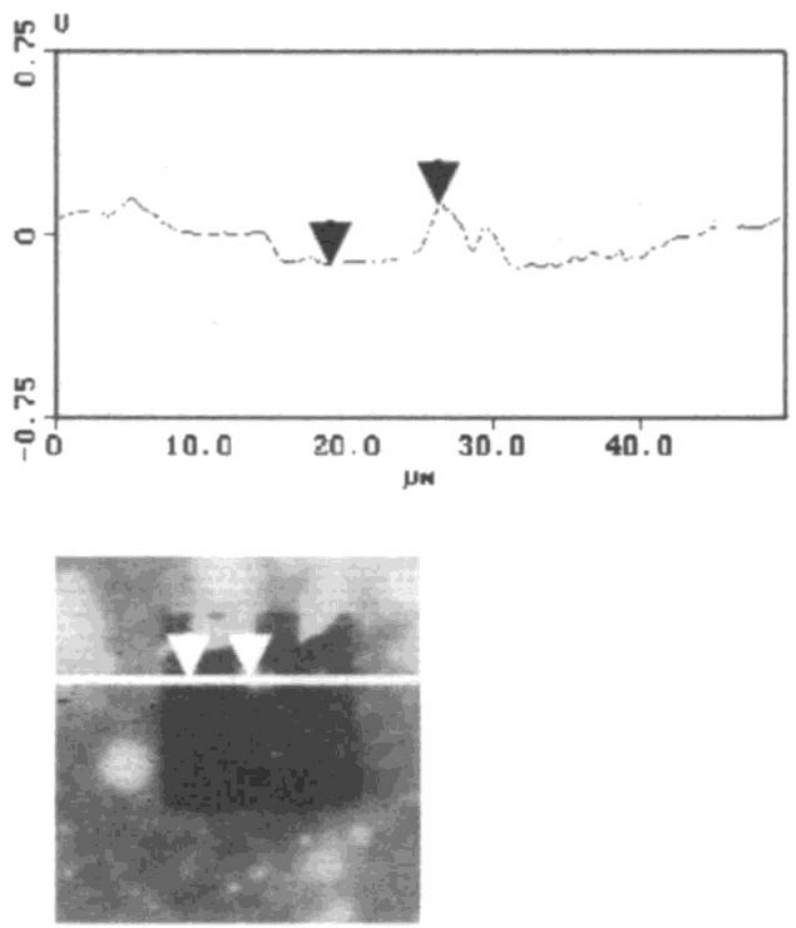

Fig. 8. Potential profile across the rastered and scratched area shown in Fig. 6. 


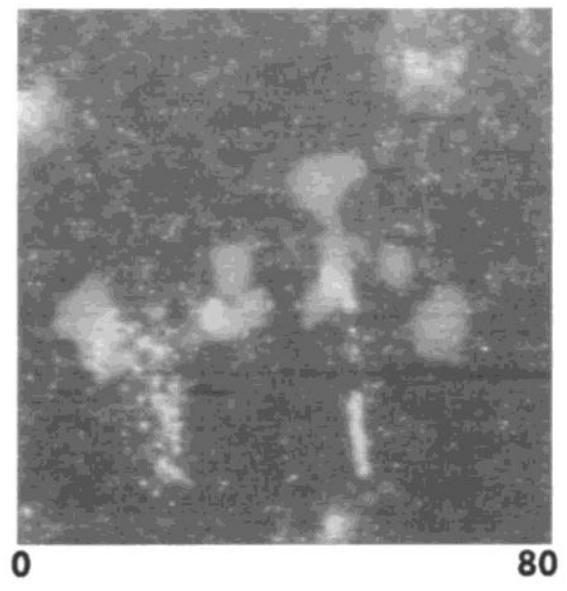

$Z$ range: $1.5 \mathrm{~V}$

um

Fig. 9. Volta potential distribution of the same area as in Fig. 2 after argon ion sputter-etching of $10 \mathrm{~nm}$ from the surface.
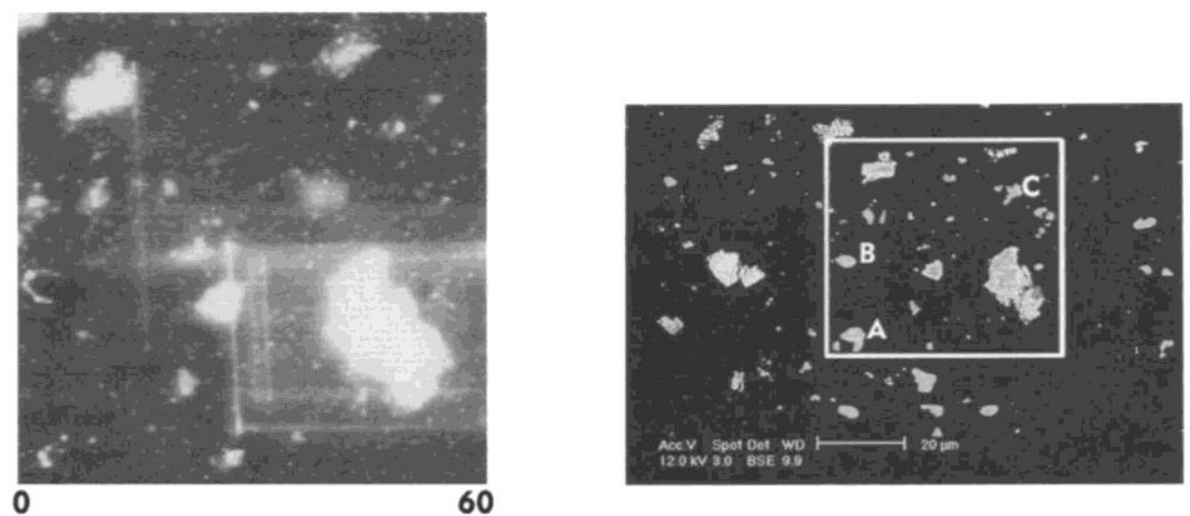

$Z$ range: IV

um

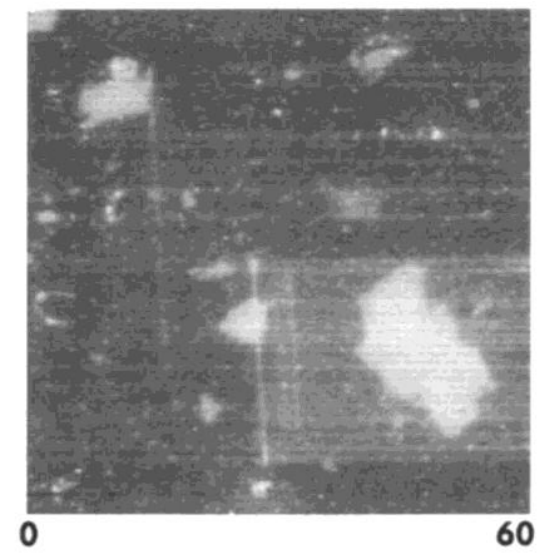

$Z$ range: IV

Fig. 10. A region of AA2024-T3 (a) potential distribution after ion sputter-etching of $10 \mathrm{~nm}$ from the surface, (b) backscattered electron image after ion sputter-etching, (c) potential distribution after 2 months in air. 
Lateral resolution. - Comments on the lateral resolution of this technique can be made by studying Fig. 10. Although submicron features containing heavier elements are visible in the backscattered image (Fig. 10b), considerably more small high-potential spots are seen in the potential map (Fig. 10a). These spots may be smaller intermetallic particles that are only revealed after etching but cannot be resolved in the SEM backscattered image. Figure 13 shows a detail of a region containing small high potential dots. Features of size ranging from 200 to 100 $\mathrm{nm}$ can easily be detected. They are not associated with visible topographical changes. Small intermetallic particles of the same size called "dispersoids" have been seen in TEM images of AA224-T3. ${ }^{3}$ The white marker in Fig. 13 indicates the position of a large contaminant particle on the surface. These particles are not associated with potential differences.

The ability to image particles of $100 \mathrm{~nm}$ in size suggests that a very high lateral resolution can be obtained with this technique. For larger particles (Fig. 2 and 3), however, the potential map indicates that the particles size is slightly larger than that shown in the backscattered electron image. The potential decrease at the edges of the intermetallic particles is also not very sharp and a transition region of $1-2 \mu \mathrm{m}$ is visible on the side (Fig. 4a and b). There are several possible explanations for the fact that the scanning Kelvin probe force microscope can detect features as small as $0.1 \mu \mathrm{m}$ in size but does not show a sharp contrast at the edges of larger particles. The potential distribution at a galvanically coupled boundary shows a similarly broad transition. ${ }^{35,36}$ This transition becomes sharper when decreasing the electrolyte thickness. In our case, adsorbed species create an extremely thin electrolyte layer of perhaps a few nanometers in thickness. Yee et al. ${ }^{19}$ mentioned that, because of the large size of the tip used for their Kelvin probe measurements, the galvanic effect is spatially narrower than the spatial resolution of the probe. In our case, because of the increased lateral resolution obtained with the AFM tip and the long range interaction of the electrostatic force, it is more difficult to attribute this phenomenon either to galvanic effect or resolution. Krause et al. ${ }^{37}$ found that for magnetic force imaging, only structures above $2 \mu \mathrm{m}$ can be truly resolved. A similar behavior is expected for electrostatic forces. There is then a transition between real potential mapping and potential contrast imaging. In this second mode, it is clear that features on the surface can be detected even if they are smaller then the real lateral resolution. The recorded potential profile has to be deconvoluted in order to have the real size and value of these small features.

Comments on the Kelvin probe technique.-A detailed explanation of the physical source of the measured potential signal determined in air and lateral resolution limitations require further study. A better understanding of the interaction of electrostatic forces at surfaces is necessary. Nonetheless, the technique is clearly a powerful tool for investigating the effects of heterogeneities in complex alloy structures. The ability to map these heterogeneities with a lateral detection limit better than $100 \mathrm{~nm}$ provides a better understanding of the corrosion behavior of the material. ${ }^{34}$ Other techniques focus on the surface composition or have limited lateral resolution. Potential is clearly a critical characteristic for corrosion studies.

The most important limitation of this technique is the fact that, as practiced now, it cannot be performed directly in solution. Even if a "solution-like" interface can be obtained outside of a bulk solution, the properties may be very different. Experiments performed on samples of AA2024-T3 in air after immersion in $\mathrm{NaCl}$ solution indicate that, under certain exposure conditions, a total loss of contrast of Volta potential at the intermetallic particles occurs as the potential of the entire surface becomes practically uniform. ${ }^{34}$ This suggests that, where it is possible, mapping of the potential in solution could also have limitations. 


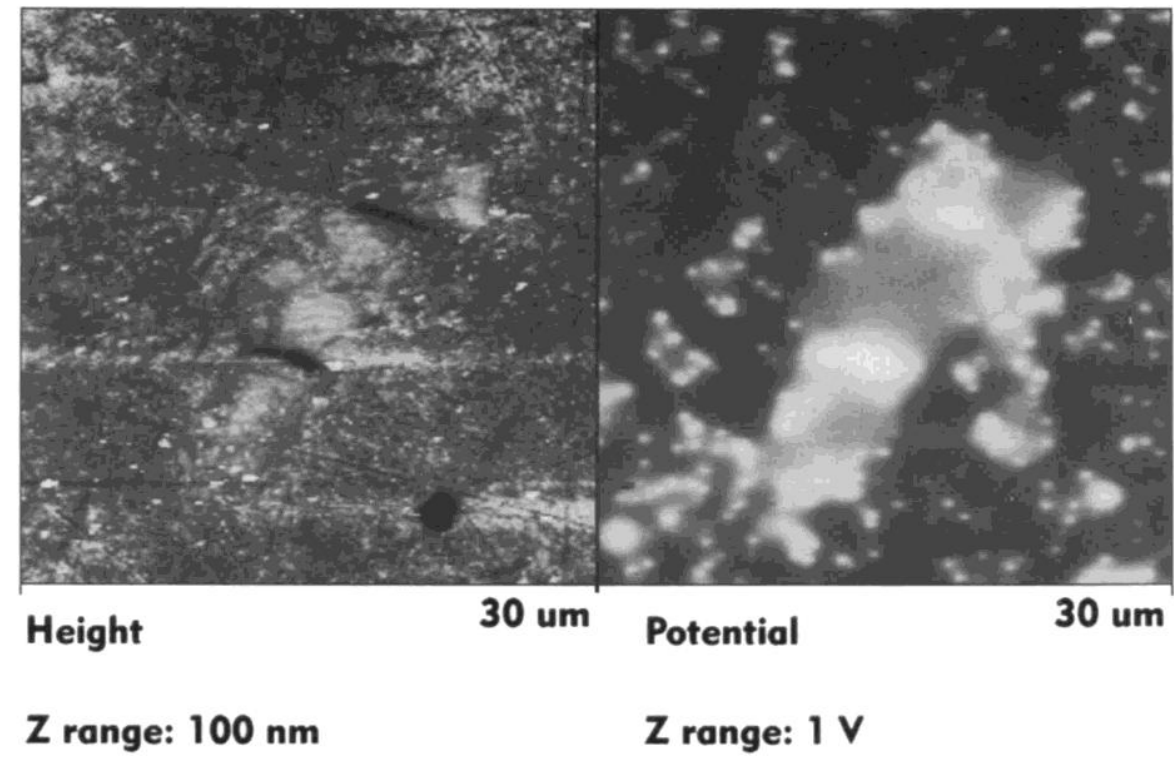

Fig. 11. Topographic and potential distribution of an $\mathrm{AI}-\mathrm{Cu}-(\mathrm{Fe}-\mathrm{Mn})$ particle after ion sputter-etching of $10 \mathrm{~nm}$ indicating a lateral heterogeneity within the particle.

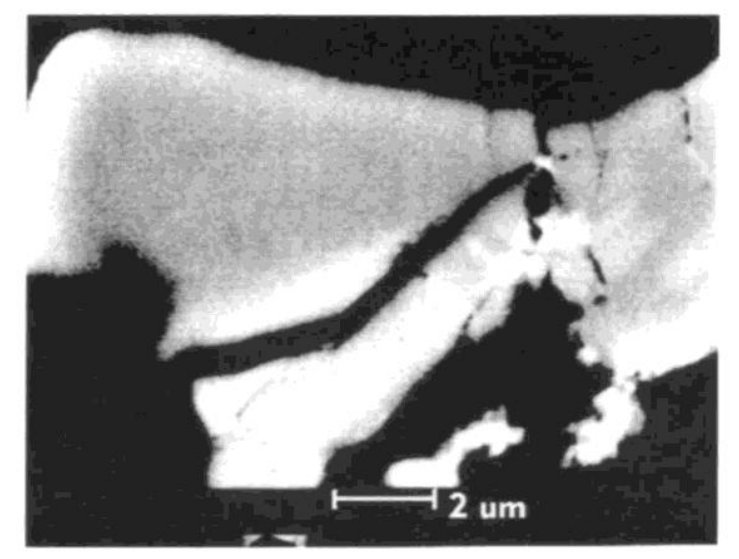

Fig. 12. Example of a backscattered electron image of an $\mathrm{Al}-\mathrm{Cu}-(\mathrm{Fe}, \mathrm{Mn})$ intermetallic particle in AA2024-T3.

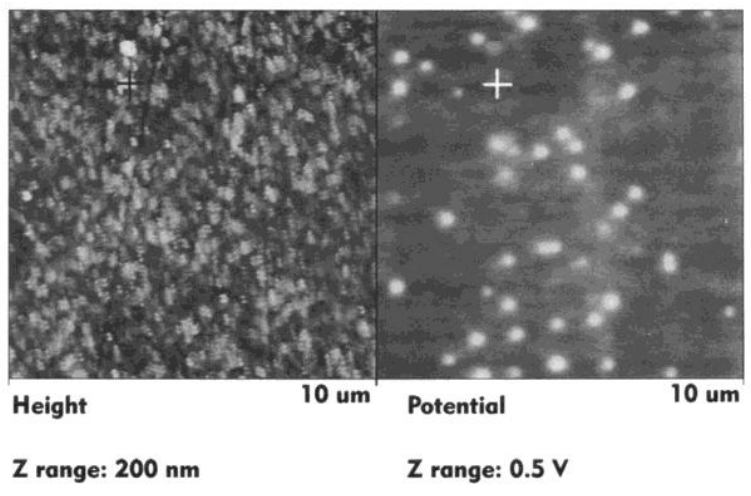

Fig. 13. Enlargement of a region in Fig. 10a with small high potential spots. 


\section{Conclusions}

The Kelvin probe method coupled with the atomic force microscope provides a unique means of obtaining a direct potential map of the surface with a submicron sensitivity for studying corrosion mechanisms. This initial study has shown how effective the Volta potential measurement can be in investigating lateral inhomogeneities on surfaces at submicron scale. In particular, the following observations were made.

1. The potential values measured in air for pure metal samples are linarly related to open-circuit potentials measured in aqueous solutions. The scanning Kelvin probe force microscope maps the Volta potential, which is a measure of the local practical nobility.

2. The intermetallic particles in AA2024-T3 show clear Volta potential contrast with respect to the matrix, which allows detailed mapping of the Volta potential distribution.

3. The noble Volta potentials obtained for $\mathrm{Al}-\mathrm{Cu}-(\mathrm{Fe}, \mathrm{Mn})$ particles are in good agreement with values measured in solution. On the other hand, the $\mathrm{Al}-\mathrm{Cu}-\mathrm{Mg}$ particles also exhibit noble Volta potentials in air, which is in contrast to active corrosion potentials reported for bulk intermetallic particles in solution. This is probably a result of the presence of a surface film. The Volta potential decreases after refreshing the surface by scratching or sputter-etching, and the Volta potential of the Al-Cu-Mg intermetallics subsequently approaches that of the matrix.

4. Lateral gradients in Volta potential are also detected in some $\mathrm{Al}-\mathrm{Cu}-(\mathrm{Fe}, \mathrm{Mn})$ intermetallic particles. These inhomogeneities have strong implications in the localized attack that can occur on and around the particles upon immersion in chloride solutions.

\section{Acknowledgments}

This work was supported by the Air Force Office of Scientific Research under contract no. F49620-96-10479, Major H. De Long, Administrator. P. S. was partially supported by the Swiss National Foundation for Research. M. Kendig, S. L. Jeanjacquet, W. Schmidt, M. Stratmann, R. Buchheit, and T. Moffat are gratefully achnowledged for providing samples and for interesting discussions.

The Ohio State University assisted in meeting the publication costs of this article.

\section{REFERENCES}

1. R. G. Buchheit, R. P. Grant, P. F Hlava, B. McKenzie, and G. L. Zender, J. Electrochem. Soc., 144, 2621 (1997).

2. C. Blanc, B. Lavelle, and G. Mankowski, Corros. Sci., 39, 495 (1997).

3. T. J. Warner, M. P. Schmidt, F. Sommer, and D. Bellot, Z. Metallkd., 86, 494 (1995).

4. B. Mazurkiewicz and A. Piotrowski, Corros. Sci., 23, 697 (1983).

5. B. Mazurkiewicz, Corros. Sci., 23, 687 (1983).

6. R. G. Buchheit, J. Electrochem. Soc., 142, 3994 (1995).

7. K. Urushino and K. Sugimoto, Corros. Sci., 19, 225 (1979).

8. I. L. Muller and J. R. Galvele, Corros. Sci., 17, 179 (1977).

9. J. R. Galvele and S. M. DeMicheli, Corros. Sci., 10, 795 (1970).

10. A. Garnier and D. Tromans, Corrosion, 35, 55 (1979).

11. K. Sugimoto, K. Hoshino, M. Kageyama, S. Kageyama, and Y. Sawada, Corros. Sci., 15, 709 (1975).

12. M. J. Pryor and J. C. Fister, J. Electrochem. Soc., 131, 1230 (1984).

13. R. Aveyard and D. A. Haydon, An Introduction of Surface Chemistry, Cambridge University Press, Cambridge (1973).

14. J. Hoelzl and F K. Schulte, in Solid Surface Physics, Vol. 85, G. Hoehler, Editor, p. 1, Springer-Verlag, Berlin 
(1979).

15. N. Kizhakevariam, I. Villegas, and M. J. Weaver, Surf. Sci., 336, 37 (1995).

16. S. Trasatti, in NATO ASI Series, Vol. 179, A. F Silva, Editor, Reidel, Dordrecht, (1986).

17. S. Trasatti, Surf. Sci., 335, 1 (1995).

18. M. Stratmann and H. Steckel, Corros. Sci., 30, 681 (1990).

19. S. Yee, R. A. Oriani, and M. Stratmann, J. Electrochem. Soc., 138, 55(1991).

20. M. Stratmann, K. T. Kim, and H. Steckel, Z. Metallkd., 81, 715(1990).

21. M. Stratmann and H. Steckel, Ber. Bunsen-Ges. Phys.Chem. I., 92, 1244 (1988).

22. R. E. Lobnig, D. J. Siconolfi, J. Maisano, G. Grundmeier, H. Steckel, R. P. Frankenthal, M. Stratmann, and J. D. Sinclair, J. Electrochem. Soc., 143, 1175 (1996).

23. Y. Martin, D. Abraham, and H. Wickramasinghe, Appl. Phys. Lett., 52, 1103 (1988).

24. C. C. Williams, J. Slinkman, W. P. Hough, and H. Wickramasinghe, Appl. Phys. Lett., 53, 1662 (1989).

25. A. Henning, J. S. T. Hochwitz, J. Never, S. Hoffmann, P. Kaszuba, and C. Daghlian, J. Appl. Phys., 77, 1888 (1995).

26. L. F. Chi, S. Jacobi, and H. Fuchs, Thin Solid Film, 284-285, 403 (1996).

27. M. Fujihira and H. Kawate, Thin Solid Films, 242,163 (1994).

28. M. Yasutake, D. Aoki, and M. Fujihjira, Thin Solid Films, 273, 279 (1996).

29. M. Fujihira, M. Sakomura, D. Aoki, and A. Koike, Thin Solid Films, 273, 168 (1996).

30. H. Yokoyama and T. Inoue, Thin Solid Films, 242, 33 (1994).

31. J. O'M. Bockris and S. U. M. Khan, Surface Electrochemistry, p. 59, Plenum Press, New York (1993).

32. D. L. Rath and D. M. Kolb, Surf. Sci., 109, 641 (1981).

33. G. J. Hansen and W N. Hansen, J. Electroanal. Chem.,150, 193 (1983).

34. P. Schmutz and G. S. Frankel, J. Electrochem. Soc.,145, 2295(1998).

35. P. Doig and P. E. J. Flewitt, J. Electrochem. Soc., 126, 2057 (1979).

36. R. Morris and W Smyrl, J. Electrochem. Soc., 136, 3229 (1989).

37. F Krause, F Kaisinger, H. Starke, G. Persch, and U. Hartmann, Thin Solid Film, 264, 141 (1995). 